\title{
Correction to: An exact scalarization method with multiple reference points for bi-objective integer linear optimization problems
}

\author{
Angelo Aliano Filho ${ }^{1}$ - Antonio Carlos Moretti ${ }^{2}$ Margarida Vaz Pato $^{3}$. \\ Washington Alves de Oliveira ${ }^{4}$
}

(c) Springer Science+Business Media, LLC, part of Springer Nature 2021

\section{Correction to: Annals of Operations Research (2021) 296:35-69 https://doi.org/10.1007/s10479-019-03317-9}

In this article a part of the context sections has errors due to an incomplete revision for which the authors much apologize.

Corrections in Definition 6 and Sect. 3.2-Theorems 1 and 3, and the penultimate sentence-are given below. These changes do not affect the remaining of the article.

Definition 6 (Supported solution and vector) A Pareto optimal solution $x^{*}$ is said to be supported if there exists $w=\left(w_{1}, w_{2}\right)^{T}>0$ such that $x^{*}$ is optimal solution of the weighted scalar problem $\min \left\{w^{T} z(x) \mid x \in X\right\}$. The resulting objective vector $z^{*}=z\left(x^{*}\right)$ is a supported non-dominated vector. $x^{*}$ and $z^{*}$ are unsupported Pareto optimal solution and unsupported non-dominated vector, respectively, if $x^{*}$ is a Pareto optimal solution and there is no $w=$ $\left(w_{1}, w_{2}\right)^{T}>0$ such that $x^{*}$ is optimal solution of the previous weighted scalar problem.

The original article can be found online at https://doi.org/10.1007/s10479-019-03317-9.

Angelo Aliano Filho

angeloaliano@utfpr.edu.br

Antonio Carlos Moretti

moretti@ime.unicamp.br

Margarida Vaz Pato

mpato@iseg.ulisboa.pt

Washington Alves de Oliveira

Washington.oliveira@fca.unicamp.br

1 Academic Department of Mathematics, Federal Technological University of Paraná, Apucarama, Brazil

2 Institute of Mathematics, Statistics and Scientific Computation, University of Campinas, Campinas, Brazil

3 ISEG and CMAFcIO, Universidade de Lisboa, Lisbon, Portugal

4 School of Applied Sciences, University of Campinas, Limeira, Brazil 


\subsection{Tchebycheff scalarization method (TCH method)}

Bowman (1976) showed a relation between the Tchebycheff norm and the Pareto frontier. For $0 \leq w \leq 1$, the TCH method is based on the minimization of the subproblem:

$$
\begin{array}{ll}
\text { Minimize } & \max \left\{w\left(z_{1}(x)-z_{1}^{-}\right),(1-w)\left(z_{2}(x)-z_{2}^{-}\right)\right\} \\
\text {subject to } & x \in X .
\end{array}
$$

The following theoretical results for Problem (2) are well known (Ehrgott 2005, p. 114).

Theorem 1 If $0<w<1$, then an optimal solution $x^{*}$ of Problem (2) is weakly Pareto optimal solution of BOILOP.

Theorem 2 If Problem (2) has a single optimal solution $x^{*}$, then $x^{*} \in X^{*}$ for BOILOP.

Theorem 3 If $x^{*} \in X^{*}$ for BOILOP, then there is a weight $0<w<1$ for which $x^{*}$ is optimal solution of Problem (2).

Note that the reference point for this method is $z^{I}$, and by selecting suitable weights $w$, Theorems 1-3 establish that all Pareto optimal solutions of BOILOP can be achieved.

Next, we present the four methods implemented in this paper. In each case, after performing an appropriate number of iterations, the Pareto filter procedure proposed by Deb and Kalyanmoy (2001) is applied to select from the list of solutions obtained only those that are Pareto optimal solutions. Each method takes advantage of the criterion space geometry to add new constraints involving the objective functions of each subproblem. Different subsets of the Pareto frontier can be obtained depending on the rule used. Moreover, the four methods achieve supported and unsupported solutions.

Publisher's Note Springer Nature remains neutral with regard to jurisdictional claims in published maps and institutional affiliations. 